\title{
ON A CLASS OF SEMILINEAR ELLIPTIC PROBLEMS NEAR CRITICAL GROWTH
}

\author{
J.V. GONCALVES * \\ Departamento de Matemática \\ Universidade de Brasilia \\ 70.910-900 Brasilia, DF, Brasil \\ S. MEIRA * \\ Vepartamento de Matemática \\ Unesp - Presidente Prudente \\ Presidente Prudente, SP, Brasil \\ (Received May 17, 1996 and in revised form January 30, 1997)

\begin{abstract}
We use Minimax Methods and explore compact embedddings in the context of Orlicz and Orlicz-Sobolev spaces to get existence of weak solutions on a class of semilinear elliptic equations with nonlinearities near critical growth. We consider both biharmonic equations with Navier boundary conditions and Laplacian equations with Dirichlet boundary conditions.
\end{abstract}

KEY WORDS AND PHRASES: Elliptic Equations, Variational Methods, Orlicz Spaces. 1991 AMS SUBJECT CLASSIFICATION CODES: 35J20, 35J25

\section{INTRODUCTION}

Our concern in this paper is on finding weak solutions for the problem

$$
(-1)^{m} \Delta^{m} u=f(x, u) \text { in } \Omega, B_{m}(u)=0 \text { on } \partial \Omega
$$

where $\Delta^{m}$ is the elliptic operator

$$
\Delta^{m} \equiv \sum_{i=1}^{N} \frac{\partial^{2 m}}{\partial x_{i}^{2 m}}+(m-1) \sum_{\substack{i, j=1, i \neq j}}^{N} \frac{\partial^{2 m}}{\partial x_{i}^{m} \partial x_{j}^{m}} \quad m=1,2,
$$

$f: \Omega \times \mathbb{R} \rightarrow \mathbb{R}$ is a Carathéodory function, $\Omega \subset \mathbb{R}^{N}$ is a bounded domain with smooth boundary $\partial \Omega$ and the boundary operator $B_{m}$ is given by

$$
B_{m}(u)=(u,(m-1) \Delta u),
$$

that is, $B_{m}(u)=0$ means either the Dirichlet or the Navier boundary conditions according to $m=1$ or $m=2$.

By a weak solution of $(1.1)$ we mean an element $u \in H_{m} \equiv H_{o}^{1}(\Omega) \cap H^{m}(\Omega)$ satisfying

$$
\langle u, v\rangle_{m}=\int_{\Omega} f(x, u) v, v \in H_{m}
$$

with $\Delta u=0$ on $\partial \Omega$ when $m=2$, where

$$
\langle u, v\rangle_{m} \equiv(m-1) \int_{\Omega} \Delta u \Delta v+(2-m) \int_{\Omega} \nabla u \nabla v u, v \in H_{m} .
$$

*Supported in Part by CNPq/Brasil. 
By the way $\langle., .\rangle_{m}$ is an inner product in $H_{m}$, we denote by $\|.\|_{m}$ its corresponding norm and we remark that $H_{m}$ is a Hilbert space.

Now let $a:[0, \infty) \rightarrow \mathbb{R}$ be a right continuous, nondecreasing function satisfying the following conditions

$$
a(0)=0, a(t)>0 \text { for } t>0, \quad a(t) \rightarrow \infty \text { as } t \rightarrow \infty
$$

and let

$$
A(t)=\int_{0}^{t} a(|s|) d s \text { and } p^{*}=\frac{2 N}{(N-2 m)} .
$$

We shall assume that both

$$
|f(x, t)| \leq C_{1}+C_{2} a(|t|), \quad(x, t) \in \Omega \times \mathbb{R}
$$

for some $C_{1} \geq 0, C_{2}>0$ and

Now consider the functional

$$
A(t)=o\left(t^{p^{*}}\right) \text { as } t \rightarrow \infty
$$

$$
I_{m}(u)=\frac{1}{2}\|u\|_{m}^{2}-\int_{\Omega} F(x, u) d x, u \in H_{m}
$$

where $F(x, t)=\int_{0}^{t} f(x, s) d s$. It follows under conditions (1.2)(1.3)(1.4) and condition (1.5) below that $I_{m} \in C^{1}\left(H_{m}, \mathbb{R}\right)$ and its derivative is given by

$$
\left\langle I_{m}^{\prime}(u), v\right\rangle=\langle u, v\rangle_{m}-\int_{\Omega} f(x, u) v \quad u, v \in H_{m} .
$$

We shall look for weak solutions of (1.1) by finding critical points of $I_{m}$. Our main result is the following.

THEOREM 1. Assume (1.2)(1.3)(1.4). Assume in addition that

$$
\begin{gathered}
a(|t|) \leq|t|^{\left(p^{*}-1\right)} \quad t \in \mathbb{R}, \\
f(x, t)=o(t) \quad t \rightarrow 0, \text { uniformly } x \in \Omega \\
0<\theta F(x, t) \leq t f(x, t) \text { a.e. } x \in \Omega \quad|t| \geq M
\end{gathered}
$$

for some $M>0, \theta>2$.

Then (1.1) has a non zero weak solution.

Our Theorem improves results by Rabinowitz [15], Gu [7], deFigueiredo, Clement \& Mitidieri [3] in the sense that we allow less restrictive growth on $f(x, t)$. It is also related to some results in Brézis \& Nirenberg [14], Pucci \& Serrin [12], van der Vorst [13].

We employ the Ambrosetti \& Rabinowitz Mountain Pass Theorem as in some of the above mentioned papers and the main point here is the use of Orlicz and Orlicz-Sobolev spaces to overcome compactness difficulties.

\section{PRELIMINARIES}

We shall apply the following variant of the Ambrosetti \& Rabinowitz [2] Mountain Pass Theorem (see Mawhin \& Willem [6]). 
THEOREM 2. Let $X$ be a Banach space and let $I \in C^{1}(X, \mathbb{R})$ with $I(0)=0$. Assume in addition that

$$
\begin{gathered}
I(u) \geq r \text { when }\|u\|=\rho, \text { for some } r, \rho>0 \\
I(e) \leq 0, \text { for some } e \in X \text { with }\|e\|>\rho .
\end{gathered}
$$

Then there is a sequence $u_{n} \in X$ such that

$$
I\left(u_{n}\right) \rightarrow c \text { and } I^{\prime}\left(u_{n}\right) \rightarrow 0
$$

where

$$
c=\inf _{\gamma \in \Gamma} \max _{0 \leq t \leq 1} I(\gamma(t)), \quad c \geq r
$$

and

$$
\Gamma=\{\gamma \in C([0,1], X) \mid \gamma(0)=0 \quad \gamma(1)=e\} .
$$

We shall apply theorem 2 with $I=I_{m}$ and $X=H_{m}$. The two lemmas below are crucial in applying theorem 2 to prove theorem 1 .

LEMMA 3. (The Mountain Pass Geometry) Assume (1.2)-(1.7). Then (2.1)-(2.2) hold true.

We remark that by lemma 3 there is a sequence $u_{n} \in H_{m}$ such that

$$
I_{m}\left(u_{n}\right) \rightarrow c \text { and } I_{m}^{\prime}\left(u_{n}\right) \rightarrow 0 .
$$

Such a sequence is called a $(P S)_{c}$ sequence.

We are going to show, (see lemma 5 below), that $u_{n}$ has a convergent subsequence. The proof of lemma 5 uses a crucial compactness type result (see lemma 4 below).

Prior to stating lemma 4 we shall recall some notations and basic results on Orlicz and OrliczSobolev spaces. We refer the reader to Krasnosels'kii \& Rutickii [5], Gossez [4], Adams [1] for an accounting on the subject. In this regard a function $A$ satisfying the set of conditions:

$$
\begin{gathered}
A \text { is convex, even, continuous } \\
A(t)=0 \text { iff } t=0 \\
\frac{A(t)}{t} \rightarrow \begin{cases}0 & \text { when } t \rightarrow 0 \\
\infty & \text { when } t \rightarrow \infty\end{cases}
\end{gathered}
$$

is referred to in the literature on Orlicz Spaces as an $\mathrm{N}$-function. An Orlicz space is defined by

$$
L_{A}(\Omega) \equiv\left\{u: \Omega \rightarrow \mathbb{R} \mid u \text { is measurable and } \int_{\Omega} A(l|u|)<\infty \text { forsome } l>0\right\}
$$

and the norm given by

$$
|u|_{A} \equiv \inf _{\alpha \in \mathbb{R}}\left\{\alpha>0 \mid \int_{\Omega} A\left(\frac{|u|}{\alpha}\right) \leq 1\right\}
$$

turns it into a (not necessarily reflexive) Banach space and as a matter of fact $L_{A}(\Omega) \rightarrow L^{1}(\Omega)$.

Corresponding to $A$ there is an $\mathrm{N}$-function labeled $\bar{A}$ called the conjugate function of $A$ which satisfies the so called Young's inequality 
and in addition

$$
s t \leq A(t)+\bar{A}(s)
$$

$$
t a(t)=A(t)+\bar{A}(a(t))
$$

where

$$
A(t)=\int_{0}^{t} a(|s|) d s
$$

and $a$ satisfies (1.2).

Moreover one also has a Hölder inequality namely

$$
\int u \cdot v \leq 2|u|_{L_{A}}|v|_{L_{A}} .
$$

Now the Orlicz-Sobolev space is defined by

$$
W^{m} L_{A}(\Omega)=\left\{u \in L_{A}(\Omega)\left|D^{\alpha} u \in L_{A}(\Omega),\right| \alpha \mid \leq m\right\}
$$

and the norm

$$
\|u\| \equiv\left[\sum_{|\alpha| \leq m}\left|D^{\alpha} u\right|_{L_{A}}^{2}\right]^{\frac{1}{2}}
$$

turns it into a Banach space.

LEMMA 4. Assume (1.4). Then $H_{m} \hookrightarrow L_{A}(\Omega), m=1,2$

LEMMA 5. Assume (1.2) - (1.7). Then the sequence $u_{n}$ has a convergent subsequence.

\section{PROOFS.}

\section{PROOF OF LEMMA 3.}

At first given $\epsilon>0$ there is by (1.6) some $\delta>0$ such that

$$
\frac{f(x, t)}{t} \leq \epsilon,|t|<\delta \quad \text { a.e. } x \in \Omega
$$

so that

$$
F(x, t) \leq \frac{\epsilon}{2} t^{2}, \quad|t|<\delta \quad \text { a.e. } x \in \Omega .
$$

On the other hand from (1.3), (1.5) we have

$$
|f(x, t)| \leq C_{1}+C_{2}|t|^{\left(p^{*}-1\right)}
$$

so that

$$
F(x, t) \leq C_{1}|t|+\frac{C_{2}}{p^{*}}|t|^{p^{*}}, \text { a.e. } x \in \Omega, \quad t \in \mathbb{R} .
$$

Hence

$$
F(x, t) \leq \frac{\epsilon}{2} t^{2}+C_{\delta}|t|^{p^{*}}, \text { a.e. } x \in \Omega, \quad t \in \mathbb{R} \text {. }
$$

Now observing that

$$
(m-1) \int_{\Omega}|\Delta u|^{2}+(2-m) \int|\nabla u|^{2} \geq \lambda_{1 m} \int_{\Omega} u^{2}
$$


where $\lambda_{1 m}$ is the first eigenvalue of

$$
\left\{\begin{array}{l}
(-1)^{m} \Delta^{m} u=\lambda u \text { in } \Omega \\
B_{m}(u)=0 \text { on } \partial \Omega
\end{array}\right.
$$

and using (3.1) we get

$$
\int_{\Omega} F(x, u) \leq \frac{\epsilon}{2 \lambda_{1 m}}|u|_{m}^{2}+C_{\delta} \int_{\Omega}|u|^{p^{*}}
$$

CLAIM 1. $|u|_{L^{p^{*}}} \leq C\|u\|_{m}, u \in H_{m}$.

Using CLAIM 1, we get

$$
\int_{\Omega} F(x, u) \leq \frac{\epsilon}{2 \lambda_{1 m}}\|u\|_{m}^{2}+C\|u\|_{m}^{p^{*}}
$$

so that

$$
I_{m}(u) \geq\left(\frac{1}{2}-\frac{\epsilon}{2 \lambda_{1 m}}\right)\|u\|_{m}^{2}-C\|u\|_{m}^{p^{*}} .
$$

Therefore there are $\rho>0, r>0$ such that

$$
I_{m}(u) \geq r, \quad\|u\|_{m}=\rho .
$$

On the other hand using (1.7) it follows that

$$
F(x, t) \geq C|t|^{\theta}, \quad|t| \geq M \text { a.e. } x \in \Omega .
$$

Now take $\phi \in C_{0}^{\infty}, \phi \geq 0, \phi \not \equiv 0$ and $\lambda>0$. Then

$$
I_{m}(\lambda \phi)=\frac{\lambda^{2}}{2}\|\phi\|_{m}^{2}-\int_{\lambda \phi \leq M} F(x, \lambda \phi)-\int_{\lambda \phi>M} F(x, \lambda \phi)
$$

Since

$$
F(x, \lambda \phi) \geq-C_{1} \lambda \phi-C_{2} A(\lambda \phi)
$$

we get

$$
\begin{aligned}
I_{m}(\lambda \phi) & \leq \frac{\lambda^{2}}{2}\|\phi\|_{m}^{2}+\int_{\lambda \phi \leq M}\left(C_{1} \lambda \phi+C_{2} A(\lambda \phi)\right)-\int_{\lambda \phi>M} F(x, \lambda \phi) \\
& \leq \frac{\lambda^{2}}{2}\|\phi\|_{m}^{2}+\int_{\lambda \phi \leq M}\left(C_{1} M+C_{2} A(M)\right)-\int_{\Omega} \phi^{\theta} \chi_{\phi>\frac{M}{\lambda}} \\
& \leq \frac{\lambda^{2}}{2}\|\phi\|_{m}^{2}+C_{M}-\lambda^{\theta} \int_{\Omega} \phi^{\theta} \chi_{\phi>\frac{M}{\lambda}} .
\end{aligned}
$$

Now, by Lebesgue Theorem

$$
\int_{\Omega} \phi^{\theta} \chi_{\phi>\frac{M}{\lambda}} \rightarrow \int_{\Omega} \phi^{\theta}
$$

Thus

$$
I_{m}(\lambda \phi) \rightarrow-\infty \text { as } \lambda \rightarrow \infty .
$$

VERIFICATION OF CLAIM 1. If $m=1$ CLAIM 1 holds by the Sobolev inequality. So let us assume $m=2$. Letting

$$
\|u\|_{2,2} \equiv \max _{|\alpha| \leq 2}\left|D^{\alpha} u\right|_{L^{2}}
$$

it is an easy matter to check that the space $H_{2}$ endowed with $\|\cdot\|_{2,2}$ is complete. We claim that

$$
\|u\|_{2} \leq C\|u\|_{2,2}
$$

Indeed, 


$$
\|u\|_{2}^{2}=\int_{\Omega}|\Delta u|^{2} \leq C\left(\max _{\imath} \int_{\Omega}\left|\frac{\partial^{2} u}{\partial x_{\imath}^{2}}\right|^{2}\right) \leq C\left(\max _{|\alpha| \leq 2}\left|D^{\alpha} u\right|_{L^{2}}^{2}\right)=C\|u\|_{2,2}^{2}
$$

Hence we also have

$$
\|u\|_{2,2} \leq C\|u\|_{2}
$$

and by Sobolev embedding we get $|u|_{L^{*}} \leq C\|u\|_{2}$, showing CLAIM 1 and thus proving lemma 3 .

The proof of lemma 4 is a consequence of a general result due to Donaldson \& Trudinger [9] (see also Adams [1, Theorem 8.40]). For the sake of completeness we recall that result in an Appendix. (see THEOREM A.1)

\section{PROOF OF LEMMA 4.}

Case $m=2$. Applying the notations of theorem A.1 let

$$
B_{0}^{-1}(t)=\sqrt{2} t^{\frac{1}{2}}, t \geq 0
$$

and

$$
\left(B_{k}\right)^{-1}(t) \equiv \int_{0}^{t} \frac{\left(B_{k-1}\right)^{-1}(\tau)}{\tau^{\frac{(N+1)}{N}}} d \tau \quad t \geq 0, \quad k=1,2
$$

We claim that

$$
\int_{1}^{\infty} \frac{\left(B_{k}\right)^{-1}(\tau)}{\tau \frac{(+1)}{N}} d \tau=\infty \text { for } k=0,1
$$

and

$$
\int_{1}^{\infty} \frac{\left(B_{k}\right)^{-1}(\tau)}{\tau \frac{(N+1)}{N}} d \tau<\infty \text { for some } k \geq 2
$$

By (3.3) and (3.4) $J$ is defined and $2 \leq J \leq N$.

Indeed by computing we find that

$$
B_{1}^{-1}(t)=\frac{\sqrt{2}(N-2)}{2 N} t^{\frac{N-2}{2 N}}
$$

and

$$
B_{2}^{-1}(t)=\frac{\sqrt{2}(N-2)}{2 N} t^{\frac{N-4}{2 N}}
$$

Now using (3.5) and (3.6) and computing again we get (3.3). Thus $J \geq 2$.

In order to show (3.4) it suffices to evaluate

$$
\int_{0}^{t} \frac{\left(B_{N-1}\right)^{-1}(\tau)}{\tau^{\frac{(N+1)}{N}}} d \tau
$$

But

and from this

$$
B_{k}^{-1}(t)=C_{N, k} t^{\frac{N-2 k}{2 N}} t \geq 0, \quad C_{N, k}>0, \quad k \geq 1
$$

$$
\int_{1}^{\infty} \frac{B_{N}^{-1}(\tau)}{\tau^{\frac{N+1}{N}}} d \tau<\infty
$$

By computing again we find that 


$$
\int_{0}^{1} \frac{\left(B_{k}\right)^{-1}(\tau)}{\tau^{\frac{(N+1)}{N}}} d \tau<\infty, \quad k=1,2 .
$$

Therefore by theorem A.1 we have

$$
W^{2} L_{B_{0}}(\Omega) \hookrightarrow L_{A}(\Omega),
$$

since as we have shown above $J \geq 2$ and yet by (1.4)

$$
\frac{B_{2}(\lambda t)}{A(t)}=\frac{C_{N, \lambda}|t|^{2^{*}}}{A(t)} \rightarrow \infty \text { as } t \rightarrow \infty, \lambda>0 .
$$

The case $m=1$ that is

$$
W^{1} L_{B_{0}}(\Omega) \hookrightarrow L_{A}(\Omega)
$$

is similar and even more direct.

Hence

$$
W^{m} L_{B_{0}}(\Omega) \hookrightarrow L_{A}(\Omega) \quad m=1,2 .
$$

Using (3.2) we finally get

$$
H_{m} \hookrightarrow L_{A}(\Omega) \quad m=1,2 .
$$

This completes the proof of lemma 4.

Before proceding to the proof of lemma 5 we consider the function $a^{*}(t) \equiv 2 C_{2} a(t)$. We remark that $a^{*}(t)$ has the same properties of $a(t)$ and in addition its potential $A^{*}(t) \equiv \int_{0}^{t} a^{*}(\tau) d \tau$ is an $\mathrm{N}$-function having the same properties as $A(t)$. In particular $A^{*}$ satisfies (1.4) and moreover

$$
|f(x, t)| \leq C_{1}+\frac{1}{2} a^{*}(t)
$$

\section{PROOF OF LEMMA 5.}

Using (1.7) we have

$$
C \geq \frac{1}{2}\|u\|_{m}^{2}-\int_{\Omega} F\left(x, u_{n}\right) \geq \frac{1}{2}\|u\|_{m}^{2}-C-\frac{1}{\theta} \int_{\Omega} u_{n} f\left(x, u_{n}\right)
$$

Now since $I_{m}^{\prime}\left(u_{n}\right) \rightarrow 0$ we have

$$
\left|\left\langle I_{m}^{\prime}\left(u_{n}\right), u_{n}\right\rangle\right| \leq \epsilon\|u\|_{m} \text { for largen }
$$

that is

Hence

$$
\|u\|_{m}^{2}-\int_{\Omega} u_{n} f\left(x, u_{n}\right) \mid \leq \epsilon\|u\|_{m} \text { for largen }
$$

$$
\begin{aligned}
C & \geq \frac{1}{2}\left\|u_{n}\right\|_{m}^{2}-C-\frac{1}{\theta}\left\|u_{n}\right\|_{m}^{2}-\frac{1}{\theta} \epsilon\left\|u_{n}\right\|_{m} \\
& =\left(\frac{1}{2}-\frac{1}{\theta}\right)\left\|u_{n}\right\|_{m}^{2}-\frac{1}{\theta} \epsilon\left\|u_{n}\right\|_{m}-C
\end{aligned}
$$

showing that $u_{n}$ is bounded in $H_{m}$. Hence by lemma 4 there is some $u \in H_{m}$ such that

$$
u_{n} \rightarrow u \text { in } H_{m} \text { and } u_{n} \rightarrow u \text { in } L_{A^{*}}(\Omega) \text {. }
$$

On the other hand, since $I_{m}^{\prime}\left(u_{n}\right) \rightarrow 0$ we have

$$
\left\langle u_{n}, \phi\right\rangle_{m}-\int_{\Omega} f\left(x, u_{n}\right) \phi=o(1), \phi \in H_{m} .
$$


We claim that

$$
\left|f\left(x, u_{n}\right)\right|_{L_{A^{*}}} \leq C, \text { for some } C>0 .
$$

Assume (3.8) for a while. Using Hölder inequality in Orlicz spaces for $L_{A^{*}}$ and $L_{\bar{A}^{*}}$ where $\bar{A}^{*}$ is the conjugate function of $A^{*}$ (see e.g. Adams [1, pg 234]) we get

$$
\left|\left\langle u_{n}, \phi\right\rangle_{m}\right| \leq o(1)+\left|f\left(x, u_{n}\right)\right|_{L_{\boldsymbol{A}^{*}}}|\phi|_{L_{A^{*}}}
$$

Now replacing $\phi$ by $u_{n}-u$ in (3.9) and using (3.8) we have

$$
0=\lim \left\langle u_{n}, u_{n}-u\right\rangle_{m}=\lim \left\langle u_{n}, u_{n}\right\rangle_{m}=\lim \left\langle u_{n}, u_{n}\right\rangle_{m}-\langle u, u\rangle_{m}
$$

showing that $u_{n} \rightarrow u$ in $H_{m}$.

VERIFICATION OF (3.8). We have

$$
\begin{aligned}
\int_{\Omega} \overline{A^{*}}\left(\left|f\left(x, u_{n}\right)\right|\right) & \leq \int_{\Omega} \overline{A^{*}}\left(C_{1}+\frac{1}{2} a^{*}\left(\left|u_{n}\right|\right)\right) \\
& \leq \frac{1}{2} \int_{\Omega} \overline{A^{*}}\left(2 C_{1}\right)+\frac{1}{2} \int_{\Omega} \overline{A^{*}}\left(a^{*}\left(\left|u_{n}\right|\right)\right) \\
& \leq C+\frac{1}{2} \int_{\Omega} A^{*}\left(\left|u_{n}\right|\right)+\int_{\Omega}\left|u_{n}\right| a^{*}\left(\left|u_{n}\right|\right) \\
& \leq C+C_{1}\left[\int_{\Omega}\left|u_{n}\right|^{p^{*}}+\int_{\Omega}\left|u_{n}\right|^{*}\right] \leq C
\end{aligned}
$$

showing (3.8) and consequently lemma 5 .

\section{PROOF OF THEOREM 1.}

We have already shown using the lemmata above that $I_{m}$ has a critical point $u \in H_{m}$ so that

$$
\langle u, v\rangle_{m}=\int_{\Omega} f(x, u) v, v \in H_{m}
$$

In the case $m=1$, we have $H_{1}=H_{o}^{1}$ and so $\mathrm{u}$ is a weak solution of $(*)_{1}$.

In the case $m=2$ it remains to show that $\Delta u=0$ on $\partial \Omega$. We use here an argument of [4].

By (1.3) and (1.5), we have

$$
f(x, u) \in{L^{p^{*}}}^{\prime}(\Omega) \text { with } \frac{1}{p^{*}}+\frac{1}{p^{*^{\prime}}}=1 .
$$

Letting $g(x)=f(x, u)$ using the fact that $p^{*}>2$ it follows that $W \equiv W^{2, p^{*}}(\Omega) \cap W_{0}^{1, p^{*}}(\Omega) \subset H_{2}$ and we have

$$
\int_{\Omega} \Delta u \Delta z=\int_{\Omega} g(x) z, \quad z \in W .
$$

Since $g(x) \in L^{p^{*^{\prime}}}(\Omega)$ there is a unique $w \in W^{2, p^{*}}(\Omega) \cap W_{0}^{1, p^{*^{\prime}}}(\Omega)$ such that

$$
\Delta w=g(x), \quad x \in \Omega \text {. }
$$

Hence

$$
\int_{\Omega} \Delta u \Delta z=\int_{\Omega} \Delta w z=\int_{\Omega} w \Delta z, \quad z \in W
$$

On the other hand given $h \in L^{p^{*}}(\Omega)$, there is a unique $z \in W$, such that

$$
\Delta z=h(x), \quad x \in \Omega
$$


Thus

$$
\int_{\Omega}(\Delta u-w) h=0, \quad h \in L^{p^{*}}(\Omega)
$$

showing that

$$
\Delta u=w \text { in } \Omega
$$

and so

$$
\Delta u=0, \text { on } \partial \Omega .
$$

This proves theorem 1 .

\section{APPENDIX}

At first we recall a general result due to Donaldson \& Trudinger [9] (see also Adams [1, theorem $8.40])$.

Let $C$ be an $\mathrm{N}$-function and consider the sequence of $\mathrm{N}$-functions

$$
\begin{gathered}
B_{0}(t) \equiv C(t), \quad t \geq 0 \\
\left(B_{k}\right)^{-1}(t) \equiv \int_{0}^{t} \frac{\left(B_{k-1}\right)^{-1}(\tau)}{\tau^{\frac{(N+1)}{N}}} d \tau, \quad k=1,2, \cdots, \quad t \geq 0 .
\end{gathered}
$$

It follows that

$$
\int_{1}^{\infty} \frac{\left(B_{k}\right)^{-1}(\tau)}{\tau^{\frac{(N+1)}{N}}} d \tau<\infty \text { for some } k \geq 1
$$

Let us label $J \equiv J(C)$ the least such $k$.

THEOREM A.1. Assume $\Omega \subset \mathbb{R}^{N}$ is a bounded domain with the cone property. Assume also that

$$
\int_{0}^{1} \frac{\left(B_{k}\right)^{-1}(\tau)}{\tau \frac{(N+1)}{N}} d \tau<\infty, \quad k=1,2, \ldots
$$

Then

$$
W^{m} L_{B_{0}}(\Omega) \rightarrow L_{B_{m}}(\Omega)
$$

provided $J \geq m$,

$$
W^{m} L_{B_{0}}(\Omega) \hookrightarrow L_{A}(\Omega)
$$

provided both $J \geq m$ and $A$ is an $\mathrm{N}$-function such that

$$
\frac{B_{m}(\lambda t)}{A(t)} \rightarrow \infty \text { as } t \rightarrow \infty, \lambda>0
$$

Next we present an example to illustrate our assumptions (1.2) - (1.5).

EXAMPLE A.2. Let $a:[0, \infty) \rightarrow \mathbb{R}$ be given by $a(t)=t^{p^{*-1}}$ if $0 \leq t<1, a(t)=$ $t^{\left(p^{*}-1\right)-\frac{1}{\log (\log (2))}}$ if $1 \leq t<3$ and $a(t)=t^{\left(p^{*}-1\right)-\frac{1}{\log (\log (n))}}$ if $n \leq t<(n+1)$ for $n=3,4, \ldots$

Then $a$ satisfies (1.2), (1.5) and it is a straightfoward calculation to show that $A$ satisfies (1.4).

\section{REFERENCES.}

[1] R. A. Adams, Sobolev spaces, Academic Press, N. York, (1987).

[2] A. Ambrosetti \& P. H. Rabinowitz, Dual variational methods in critical point theory and applications, J. Funct Anal 14 (1973) 349 - 381. 
[3] D. G de Figueiredo \& Ph. Clement \& E. Mitidieri, Positive solutions of semilinear elliptic problems, Comm. P.D.E. 17 (1992), 932-940

[4] J. P. Gossez, Orlicz Spaces, Orlicz-Sobolev Spaces and strongly nonlinear elliptc problems, Univ. de Brasília (1976).

[5] M. A. Krasnosel'skii \& Ya. B. Rutickii, Convex functions and Orlicz spaces, New York (1961).

[6] J. Mawhin \& M. Willem, Critical point theory and Hamiltonian systems, Appl. Math. Sc 74 Springer-Verlag (1989).

[7] Yong-Geng Gu, Nontrivial solutions of semilinear elliptic equations of fourth order, Proc. of Symposia in Pure Math. 45 (1986) Part I.

[8] N. S. Trudinger, On imbedding into Orlicz spaces and some applications, J. Math. Mech. 17 (1967) 473-484.

[9] T. K. Donaldson \& N. S. Trudinger, Orlicz-Sobolev spaces and imbedding, J. of Funct. Anal. 8 (1971).

[10] J. A. Hempel, G. R. Morris \& N. S. Trudinger, On the sharpness of a limiting case of the Sobolev imbedding theorem, Bull. Austral. Math. Soc. 3 (1970) 369-373. 333-336.

[11] D. Gilbarg \& N. Trudinger, Elliptic partıal differential equations of second order, SprigerVerlag, Berlin (1977) 455-477.

[12] P. Pucci \& J. Serrin, Critical exponents and critical dimensions for polyharmonic operators, J. Math Pures et Appl., 69 (1990) 55-83.

[13] R. C. A. M. van der Vorst, Variational identities and applicatıons to differential systems, Arch Rational Mech. Anal., 116 (1991) 375-398.

[14] H. Brézis \& L. Nirenberg, Positıve solutions of nonlinear elliptic equations involving critical Sobolev exponents. Comm. Pure App. Math. XXXVI (1983) 437-477.

[15] P. H. Rabinowitz, Some minimax theorems and app. to nonlinear PDE, In Nonl. Anal. (Ed. Cesari, Kannan \& Weinberger), Acad. Press (1978). 


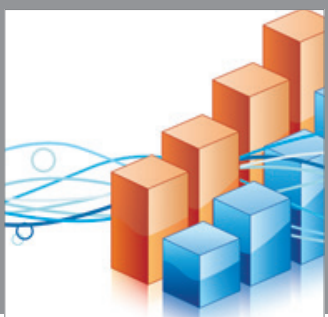

Advances in

Operations Research

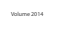

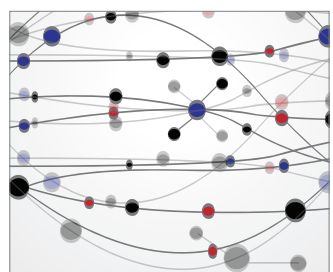

\section{The Scientific} World Journal
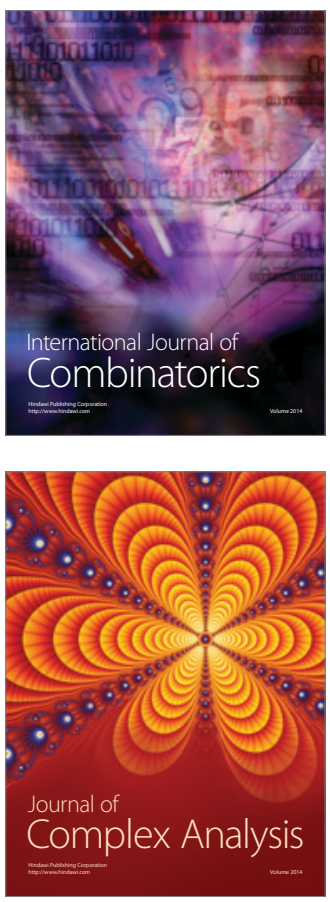

International Journal of

Mathematics and

Mathematical

Sciences
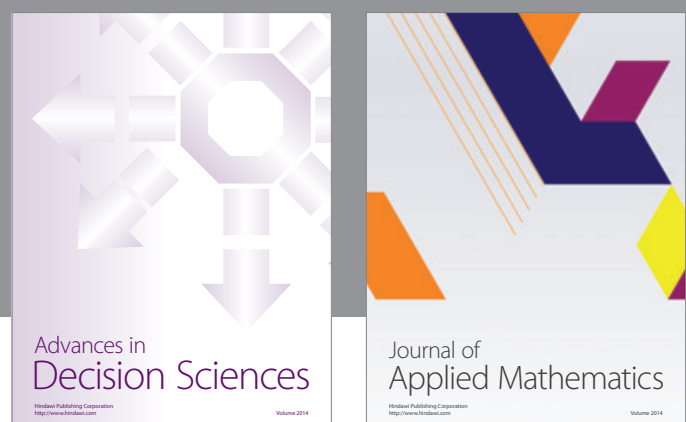

Journal of

Applied Mathematics
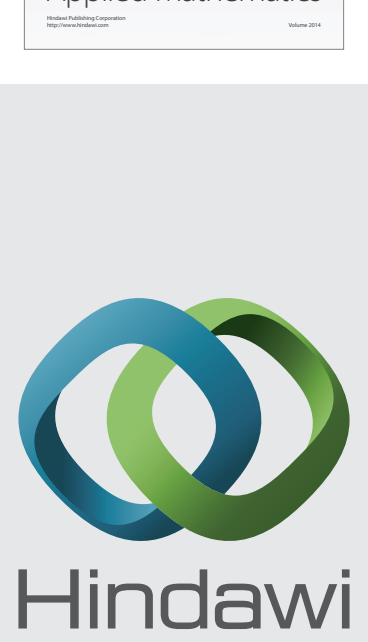

Submit your manuscripts at http://www.hindawi.com
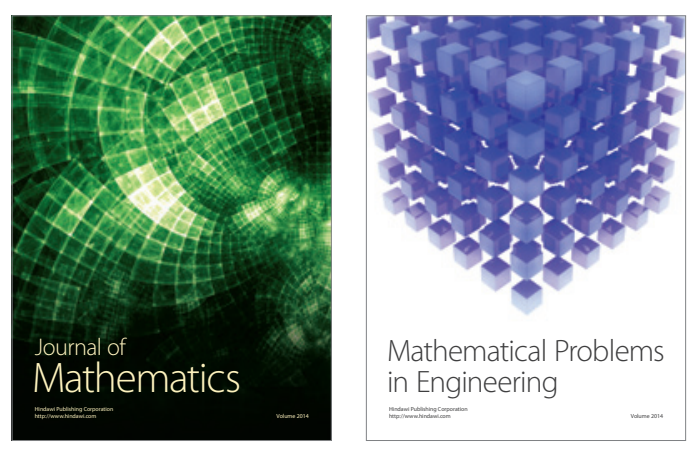

Mathematical Problems in Engineering
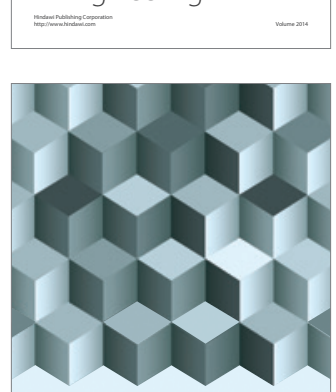

Journal of

Function Spaces
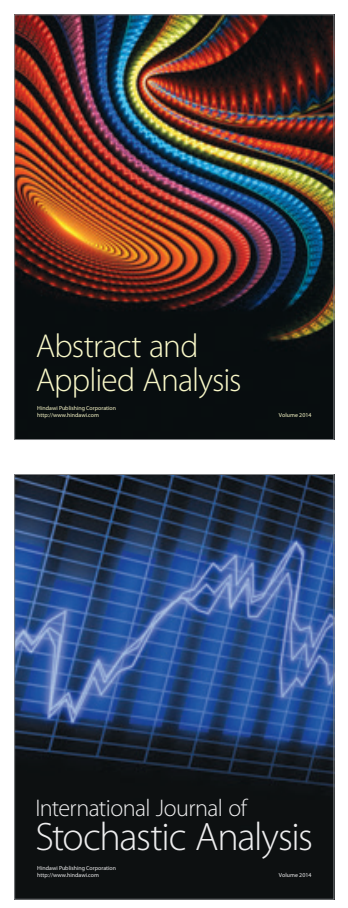

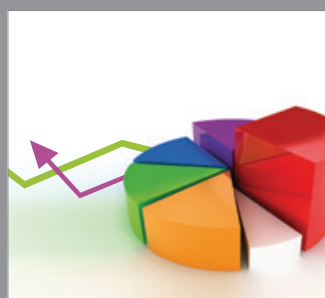

ournal of

Probability and Statistics

Promensencen
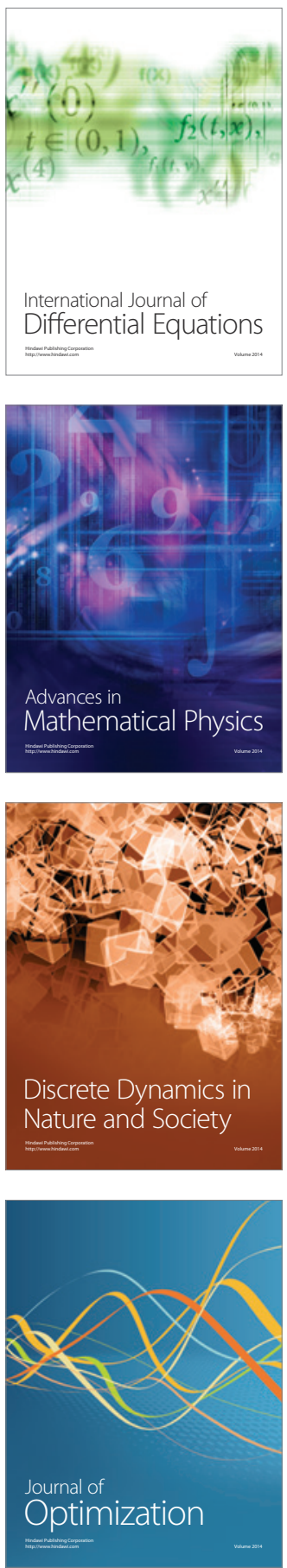